Proceedings of the Edinburgh Mathematical Society (2005) 48, 37-49 (C)

DOI:10.1017/S0013091504000094 Printed in the United Kingdom

\title{
ELEMENTARY OPERATORS AS LIE HOMOMORPHISMS OR COMMUTATIVITY PRESERVERS
}

\author{
MATEJ BREŠAR ${ }^{1}$ AND PETER ŠEMRL ${ }^{2}$ \\ ${ }^{1}$ Department of Mathematics, University of Maribor, PEF, Koroška 160, \\ SI-2000 Maribor, Slovenia (bresar@uni-mb.si) \\ ${ }^{2}$ Department of Mathematics, University of Ljubljana, Jadranska 19, \\ SI-1000 Ljubljana, Slovenia (peter.semrl@fmf.uni-lj.si)
}

(Received 16 February 2004)

\begin{abstract}
We consider elementary operators on centrally closed prime algebras that are Lie (or Jordan) homomorphisms or commutativity preservers.

Keywords: elementary operator; Lie homomorphism; Jordan homomorphism; commutativity preserver; centrally closed prime algebra

2000 Mathematics subject classification: Primary 16N60

Secondary 16R50; 47B47
\end{abstract}

\section{Introduction}

Let $\mathcal{A}$ be an algebra and let $\phi: \mathcal{A} \rightarrow \mathcal{A}$ be a linear map. Recall that $\phi$ is said to be a Lie homomorphism if $\phi([X, Y])=[\phi(X), \phi(Y)]$ for all $X, Y \in \mathcal{A}$. Here, $[X, Y]$ denotes the commutator $X Y-Y X$ of $X$ and $Y$. More generally, we say that $\phi$ preserves commutativity if for all $X, Y \in \mathcal{A},[X, Y]=0$ implies $[\phi(X), \phi(Y)]=0$. The standard problem is to describe the form of these maps; the usual conclusion is that they are close to (anti)homomorphisms.

The studies of both types of map have a long history. The one on Lie homomorphisms has its roots in the theory of Lie algebras $[\mathbf{1 5}]$ and ring theory $[\mathbf{1 3}, \mathbf{1 4}]$, while the more general problem of determining commutativity preservers begun in linear algebra $[\mathbf{2 2}]$. Over the years these maps have been considered in various areas. We refer, however, only to a few recent publications, where one can find further references and historical accounts $[\mathbf{2}, \mathbf{4}, \mathbf{5}, \mathbf{8}, \mathbf{2 1}]$.

In order to describe a Lie homomorphism or a commutativity preserver $\phi$, one is usually forced to impose some conditions on the range of $\phi$. An exception is the recent result from $[\mathbf{2 1}]$ which states that an arbitrary linear commutativity preserver on the matrix algebra $\mathcal{A}=M_{n}(\mathbb{F}), \mathbb{F}$ an algebraically closed field of characteristic 0 , has either a commutative range or it is of a standard form (which can be described via (anti)automorphisms). 
The same result definitely does not hold true in some natural infinite-dimensional generalizations of $M_{n}(\mathbb{F})$ (like $\mathcal{B}(H)$ ), and, in fact, it seems out of reach to describe precisely entirely arbitrary commutativity preservers on these algebras. We will therefore confine ourselves to a certain class of linear operators, the so-called elementary operators. Recall that $\phi: \mathcal{A} \rightarrow \mathcal{A}$ is called an elementary operator on $\mathcal{A}$ if there exist $A_{i}, B_{i} \in \mathcal{A}$ such that

$$
\phi=\sum_{i=1}^{n}{ }_{A_{i}} M_{B_{i}}
$$

where ${ }_{A} M_{B}: T \mapsto A T B$. It is easy to check (see below) that every linear operator on $M_{n}(\mathbb{F}), \mathbb{F}$ a field, is an elementary operator. Therefore, elementary operators on infinitedimensional algebras can be considered as natural generalizations of linear operators on matrix algebras.

The methods that we will use are somewhat different from those that are usual in the study of Lie homomorphisms and commutativity preservers. All concepts and tools that we need will be briefly surveyed in $\S 2$. When dealing with elementary operators it is natural to restrict the attention to prime algebras. Because otherwise ${ }_{A} M_{B}$ can be 0 for some non-zero $A, B \in \mathcal{A}$, which makes the treatment of general elementary operators rather muddled. In order to avoid various technicalities (and thereby trying to make the paper interesting to a wider audience), we will consider only centrally closed prime algebras. Bijective commutativity preservers (as well as Lie isomorphisms) on such algebras were described in $[6]$. As we will see, replacing the bijectivity assumption with the assumption that a map is an elementary operator yields quite different conclusions. The Lie homomorphism problem will be treated in $\S 3$. An almost identical argument also works for Jordan homomorphisms, i.e. linear maps $\phi: \mathcal{A} \rightarrow \mathcal{A}$ satisfying $\phi(X Y+Y X)=$ $\phi(X) \phi(Y)+\phi(Y) \phi(X)$ for all $X, Y \in \mathcal{A}$. The problem of describing elementary operators that preserve commutativity, which is treated in $\S 4$, is considerably more difficult and the result that we obtain is less definitive than in the Lie (or Jordan) homomorphism case.

\section{Preliminaries}

\subsection{Elementary operators}

Elementary operators appear in many branches of mathematics; however, they have been systematically studied mostly in operator theory. We refer the reader to the recent treatise [2, Chapter 5].

As already mentioned, every linear operator on $M_{n}(\mathbb{F})$ is an elementary operator. Indeed, just note that the set $\left\{E_{i j} M_{E_{k l}}: i, j, k, l=1, \ldots, n\right\}$ (here, $E_{i j}$ denotes a matrix unit) is a linearly independent subset of the algebra of all linear operators on $M_{n}(\mathbb{F})$, and, hence, since its cardinality is $n^{4}$, it is a basis of this algebra.

Let us introduce some terminology. Given an elementary operator $\phi=\sum_{i=1}^{n} A_{i} M_{B_{i}}$, we will call the elements $A_{1}, \ldots, A_{n}$ the left coefficients of $\phi$, and, similarly, the $B_{j}$ will be called the right coefficients of $\phi$. If $\phi$ cannot be represented as $\phi=\sum_{j=1}^{k} C_{j} M_{D_{j}}$ for 
some $k<n$, then we say that $\phi$ is an elementary operator of length $n$. The zero operator has length 0 .

\subsection{Centrally closed prime algebras}

Let $\mathcal{A}$ be a prime unital algebra over a field $\mathbb{F}$. We say that $\mathcal{A}$ is centrally closed (over $\mathbb{F}$ ) if $\mathbb{F}$ coincides with the extended centroid of $\mathcal{A}$. In our arguments we will just apply some properties of centrally closed prime algebras, so we will not deal with the extended centroid directly. Therefore, we will not give its exact definition here; let us just say that the extended centroid of a prime algebra is a certain field containing (an isomorphic copy of) the centre of the algebra (so, if $\mathcal{A}$ is centrally closed over $\mathbb{F}$, then the centre of $\mathcal{A}$ is equal to $\mathbb{F} \mathbf{1})$. For details we refer to [3, Chapter 2]. Let us list some important examples of centrally closed prime algebras: these are unital central simple algebras, the coproducts of two algebras of certain non-trivial dimensions [18], primitive Banach algebras (this follows, for example, from [3, Corollary 4.1.2]), and ultraprime normed algebras [19]. The latter class of algebras includes, for example, prime $C^{*}$-algebras, standard operator algebras, and prime group algebras $l^{1}(G)$, where $G$ is a discrete group [23].

We will need the following lemma which is essentially due to Martindale [17]. The version which we will state, however, follows from [16, Lemma 2.1].

Lemma 2.1. Let $\mathcal{A}$ be a centrally closed prime algebra over $\mathbb{F}$. Further, let $A_{i}, B_{i} \in \mathcal{A}$ and let $\phi=\sum_{i=1}^{n} A_{i} M_{B_{i}}$. Suppose that for some $k, 1 \leqslant k \leqslant n$, the elements $A_{1}, \ldots, A_{k}$ are linearly independent over $\mathbb{F}$. If $\phi=0$, then for every $i, 1 \leqslant i \leqslant k$, there exist $\lambda_{i j} \in \mathbb{F}$, $k+1 \leqslant j \leqslant n$, such that $B_{i}+\sum_{j=k+1}^{n} \lambda_{i j} B_{j}=0$ (in particular, each $B_{i}=0$ in the case $k=n)$.

Corollary 2.2. Let $\mathcal{A}$ be a centrally closed prime algebra over $\mathbb{F}$. An elementary operator $\phi=\sum_{i=1}^{n} A_{i} M_{B_{i}}$ has length $n$ if and only if the sets $\left\{A_{1}, \ldots, A_{n}\right\}$ and $\left\{B_{1}, \ldots, B_{n}\right\}$ are linearly independent.

Proof. Suppose that $\phi$ can be represented as $\phi=\sum_{j=1}^{k} C_{j} M_{D_{j}}$. Thus,

$$
\sum_{i=1}^{n}{ }_{A_{i}} M_{B_{i}}-\sum_{j=1}^{k}{ }_{C_{j}} M_{D_{j}}=0 .
$$

Assuming that the $A_{i}$ are linearly independent, it follows from Lemma 2.1 that each $B_{i}$, $i=1, \ldots, n$, lies in the linear span of $\left\{D_{1}, \ldots, D_{k}\right\}$. If the $B_{i}$ are also linearly independent, then we must have $k \geqslant n$. Therefore, $\phi$ has length $n$ in this case. Conversely, assuming, for example, that $A_{1}$ is a linear combination of $A_{2}, \ldots, A_{n}$, it follows immediately that the length of $\phi$ is less than or equal to $n-1$.

\subsection{The (lower) socle}

Let $\mathcal{A}$ be a semiprime algebra. Recall that the socle of $\mathcal{A}$ is the sum of all minimal left ideals of $\mathcal{A}$. If $\mathcal{A}$ does not have minimal left ideals, then we define that the socle of $\mathcal{A}$ is 0 . It turns out that the socle coincides with the sum of all minimal right ideals of $\mathcal{A}$, and 
so it is an ideal of $\mathcal{A}$. Every minimal left ideal $\mathcal{L}$ of $\mathcal{A}$ is of the form $\mathcal{L}=\mathcal{A} E$, where $E$ is a minimal idempotent in $\mathcal{A}$, that is, an idempotent such that $E \mathcal{A} E$ is a division algebra. We will also make use of the concept introduced in the recent paper [9]: the lower socle, which we denote by $\mathcal{S}$, of a semiprime algebra $\mathcal{A}$ is the sum of all minimal left ideals $\mathcal{A} E$ such that the division algebra $E \mathcal{A} E$ is finite dimensional (or 0 if there are no such minimal idempotents). It turns out that $\mathcal{S}$ is also an ideal of $\mathcal{A}$. In the case where $\mathcal{A}$ is prime, $\mathcal{S}$ either coincides with the socle or it is 0 .

We will need two results from [9]. The first one is $[\mathbf{9}$, Theorem 3.3].

Lemma 2.3. Let $\mathcal{A}$ be a semiprime algebra. Then $A \in \mathcal{S}$ if and only if the operator ${ }_{A} M_{A}$ has finite rank.

We will in fact use only a very special case of this lemma, and moreover its easier 'only if' part. The second result is a slightly sharpened version of [9, Corollary 4.3].

Lemma 2.4. Let $\mathcal{A}$ be a centrally closed prime algebra over $\mathbb{F}$. Further, let $A_{i}, B_{i} \in \mathcal{A}$ and let $\phi=\sum_{i=1}^{n} A_{i} M_{B_{i}}$. Suppose that for some $k, 1 \leqslant k \leqslant n$, the elements $A_{1}, \ldots, A_{k}$ are linearly independent over $\mathbb{F}$. If $\phi$ has finite rank, then for every $i, 1 \leqslant i \leqslant k$, there exist $\lambda_{i j} \in \mathbb{F}, k+1 \leqslant j \leqslant n$, such that $B_{i}+\sum_{j=k+1}^{n} \lambda_{i j} B_{j} \in \mathcal{S}$ (in particular, each $B_{i} \in \mathcal{S}$ in the case $k=n$ ).

Proof. For $k=n$ the result follows from [9, Theorem 4.2]. So, let $1 \leqslant k<n$. We may assume without loss of generality that $\left\{A_{1}, \ldots, A_{k}\right\}$ is a maximal linearly independent subset of $\left\{A_{1}, \ldots, A_{n}\right\}$. Therefore, for every $j, k+1 \leqslant j \leqslant n$, we have $A_{j}=\sum_{i=1}^{k} \lambda_{i j} A_{i}$ for some $\lambda_{i j} \in \mathbb{F}$, which implies that $\phi=\sum_{i=1}^{k} A_{i} M_{D_{i}}$, where $D_{i}=B_{i}+\sum_{j=k+1}^{n} \lambda_{i j} B_{j}$. Since the $A_{i}$ are linearly independent, we can use $[\mathbf{9}$, Theorem 4.2] to conclude that each $D_{i}$ lies in $\mathcal{S}$. This is the desired conclusion.

\subsection{Prime GPI algebras}

An algebra $\mathcal{A}$ is said to be a GPI algebra if it satisfies a non-zero generalized polynomial identity. Very informally, this means that arbitrary elements in $\mathcal{A}$ satisfy an identity which involves some fixed elements in $\mathcal{A}$ (for example, if $E$ is a minimal idempotent such that $E \mathcal{A} E=\mathbb{F} E$, then $E X E Y E=E Y E X E$ for all $X, Y \in \mathcal{A}$, which can be viewed as a nonzero generalized polynomial identity). For details, however, we refer to [3, Chapter 6].

Let $\mathcal{A}$ be a centrally closed prime algebra over $\mathbb{F}$. The celebrated theorem by Martindale [17] (see also [3, Theorem 6.1.6]) then tells us that $\mathcal{A}$ is a GPI algebra if and only if it has a non-zero lower socle. Assume that $\mathbb{F}$ is algebraically closed. If $E$ is a minimal idempotent $\mathcal{A}$, then $E \mathcal{A} E$, as a finite-dimensional division algebra over $\mathbb{F}$, must be one dimensional, so that $E \mathcal{A} E=\mathbb{F} E$. By [3, Theorem 4.3.9] there exists a pair of dual vector spaces $\mathcal{V}$ and $\mathcal{W}$ over $\mathbb{F}$ such that $\mathcal{F}_{W}(\mathcal{V}) \subseteq \mathcal{A} \subseteq \mathcal{L}_{\mathcal{W}}(\mathcal{V})$. Let us explain the meaning of this notation and terminology. We say that $\mathcal{V}$ and $\mathcal{W}$ are a pair of dual spaces if there is a bilinear form $\langle\cdot, \cdot\rangle: \mathcal{V} \times \mathcal{W} \rightarrow \mathbb{F}$ which is non-degenerate (i.e. $\langle v, \mathcal{W}\rangle=0$ implies $v=0$ and $\langle\mathcal{V}, w\rangle=0$ implies $w=0$ ). A linear map $A: \mathcal{V} \rightarrow \mathcal{V}$ is said to have an adjoint $A^{*}: \mathcal{W} \rightarrow \mathcal{W}$ if $\langle A v, w\rangle=\left\langle v, A^{*} w\right\rangle$ for all $v \in \mathcal{V}, w \in \mathcal{W}$. For example, a rank-one operator $v \mapsto\left\langle v, w_{0}\right\rangle v_{0}$ has the adjoint $w \mapsto\left\langle v_{0}, w\right\rangle w_{0} . \mathcal{L}_{\mathcal{W}}(\mathcal{V})$ denotes the 
algebra of all linear operators on $\mathcal{V}$ that have an adjoint, and $\mathcal{F}_{W}(\mathcal{V})$ denotes the algebra of all linear operators on $\mathcal{V}$ that have an adjoint and are of finite rank. Let us point out that $A \in \mathcal{L}_{\mathcal{W}}(\mathcal{V})$ has finite rank, i.e. it lies in $\mathcal{F}_{W}(\mathcal{V})$, if and only if $A^{*}$ has finite rank. One can prove this easily by making use of the following result [3, Theorem 4.3.1]: if $v_{1}, \ldots, v_{n} \in \mathcal{V}$ are linearly independent, then there exist $w_{1}, \ldots, w_{n} \in \mathcal{W}$ such that $\left\langle v_{i}, w_{j}\right\rangle=\delta_{i j}, i, j=1, \ldots, n$. We also mention that $\mathcal{F}_{W}(\mathcal{V})$ is equal to the lower socle of $\mathcal{A}[\mathbf{3}$, Theorem 4.3.8].

\subsection{Generalized functional identities}

Roughly speaking, the theory of generalized functional identities deals with identities satisfied by arbitrary elements from a ring, which involve arbitrary maps and some fixed elements from a ring. An introductory account on this topic can be found in the survey [7]. We will need only a rather elementary result of this theory which is due to Chebotar [11, Theorems 2.6 and 2.7]. We will state only its simplified version, which is sufficient for our purposes.

Lemma 2.5. Let $\mathcal{A}$ be a centrally closed prime algebra, let $A_{1}, \ldots, A_{n}$ be linearly independent elements in $\mathcal{A}$, let $r \geqslant 1$ and let $f_{i j}: \mathcal{A}^{r-1} \rightarrow \mathcal{A}$ be arbitrary maps. Suppose that either

$$
\sum_{i=1}^{r} \sum_{j=1}^{n} A_{j} X_{i} f_{i j}\left(X_{1}, \ldots, X_{i-1}, X_{i+1}, \ldots, X_{r}\right)=0
$$

for all $X_{1}, \ldots, X_{r} \in \mathcal{A}$, or

$$
\sum_{i=1}^{r} \sum_{j=1}^{n} f_{i j}\left(X_{1}, \ldots, X_{i-1}, X_{i+1}, \ldots, X_{r}\right) X_{i} A_{j}=0
$$

for all $X_{1}, \ldots, X_{r} \in \mathcal{A}$. Then either each $f_{i j}=0$ or $\mathcal{A}$ is a GPI algebra.

\subsection{Locally linearly dependent operators}

Let $\mathcal{U}$ and $\mathcal{V}$ be vector spaces and let $\mathcal{V}_{0}$ be a subspace of $\mathcal{V}$. We say that linear operators $A_{1}, \ldots, A_{n}: \mathcal{U} \rightarrow \mathcal{V}$ are locally linearly dependent modulo $\mathcal{V}_{0}$ if for every $u \in \mathcal{U}$ there exists a non-trivial linear combination of $A_{1} u, \ldots, A_{n} u$ that is contained in $\mathcal{V}_{0}$. We will need only the fundamental lemma on such operators, which is due to Amitsur [1, Lemma 1] (incidentally, we remark that one can find various generalizations in the recent papers $[\mathbf{1 0}]$ and $[\mathbf{2 0}])$.

Lemma 2.6. If operators $A_{1}, \ldots, A_{n}$ are locally linearly dependent modulo some finite-dimensional space $\mathcal{V}_{0}$, then there exists a non-trivial linear combination of $A_{1}, \ldots, A_{n}$ of finite rank.

\section{Elementary operators that are Lie (Jordan) homomorphisms}

Theorem 3.1. Let $\mathcal{A}$ be an infinite-dimensional centrally closed prime algebra, and let $\phi=\sum_{i=1}^{n} A_{i} M_{B_{i}}$ be an elementary operator on $\mathcal{A}$ of length $n$. The following conditions are equivalent: 
(i) $\phi$ is a Lie homomorphism;

(ii) $\phi$ is a Jordan homomorphism;

(iii) $\phi$ is a homomorphism;

(iv) $B_{i} A_{j}=\delta_{i j}, i, j=1, \ldots, n$.

Proof. It is clear that (iv) implies (iii), and that (iii) implies both (i) and (ii). Therefore, it suffices to show that each of (i) and (ii) implies (iv). We will only prove that (i) implies (iv), since the proof that (ii) implies (iv) is almost identical.

So let $\phi$ be a Lie homomorphism. We have $\phi(X Y-Y X)=\phi(X) \phi(Y)-\phi(Y) \phi(X)$ for all $X, Y \in \mathcal{A}$, that is,

$$
\sum_{i=1}^{n} A_{i} X Y B_{i}-\sum_{i=1}^{n} A_{i} Y X B_{i}=\sum_{i=1}^{n} \sum_{j=1}^{n} A_{i} X B_{i} A_{j} Y B_{j}-\sum_{i=1}^{n} \sum_{j=1}^{n} A_{j} Y B_{j} A_{i} X B_{i} .
$$

We can rewrite this as

$$
\sum_{i=1}^{n} A_{i} X\left(\sum_{j=1}^{n}\left(\delta_{i j}-B_{i} A_{j}\right) Y B_{j}\right)+\sum_{i=1}^{n}\left(\sum_{j=1}^{n} A_{j} Y\left(B_{j} A_{i}-\delta_{i j}\right)\right) X B_{i}=0 .
$$

Since $\phi$ has length $n, A_{1}, \ldots, A_{n}$ are linearly independent (Corollary 2.2). Therefore, it follows from Lemma 2.1 that for every $i, 1 \leqslant i \leqslant n$, and every $Y \in \mathcal{A}$, $\sum_{j=1}^{n}\left(\delta_{i j}-B_{i} A_{j}\right) Y B_{j}$ is a linear combination of $B_{1}, \ldots, B_{n}$. That is to say, the elementary operator

$$
\sum_{j=1}^{n}\left(\delta_{i j}-B_{i} A_{j}\right) M_{B_{j}} \text { has finite rank for every } i=1, \ldots, n .
$$

Since $B_{1}, \ldots, B_{n}$ are also linearly independent it follows from (the symmetric version of) Lemma 2.4 that

$$
\delta_{i j}-B_{i} A_{j} \in \mathcal{S}, \quad i, j=1, \ldots, n,
$$

where $\mathcal{S}$ denotes the lower socle of $\mathcal{A}$.

Suppose that $\mathbf{1}-B_{1} A_{1} \neq 0$. Again applying Lemma 2.4 we infer from (3.1) that $B_{1}+\sum_{i=2}^{n} \lambda_{i} B_{i} \in \mathcal{S}$ for some $\lambda_{i} \in \mathbb{F}$. Consequently, $B_{1} A_{1}+\sum_{i=2}^{n} \lambda_{i} B_{i} A_{1} \in \mathcal{S}$. However, by (3.2), $B_{i} A_{1} \in \mathcal{S}, i \neq 1$, and $\mathbf{1}-B_{1} A_{1} \in \mathcal{S}$, and so it follows that $\mathbf{1} \in \mathcal{S}$. But then Lemma 2.3 tells us that $\mathcal{A}={ }_{\mathbf{1}} M_{\mathbf{1}}(\mathcal{A})$ is finite dimensional, a contradiction. Therefore, $B_{1} A_{1}=\mathbf{1}$. Similarly, $B_{i} A_{i}=\mathbf{1}$ for every $i$.

Suppose that $B_{1} A_{2} \neq 0$. Since $B_{1} A_{1}=\mathbf{1}$, for $i=1$ the assertion (3.1) now reduces to the conclusion that $\sum_{j=2}^{n} B_{1} A_{j} M_{B_{j}}$ has finite rank. But then it follows from Lemma 2.4 that $B_{2}+\sum_{i=3}^{n} \mu_{i} B_{i} \in \mathcal{S}$ for some $\mu_{i} \in \mathbb{F}$. Hence $B_{2} A_{2}+\sum_{i=3}^{n} \mu_{i} B_{i} A_{2} \in \mathcal{S}$. However, since $B_{2} A_{2}=\mathbf{1}$ and $B_{i} A_{2} \in \mathcal{S}, i \geqslant 3$, we again arrive at the contradiction that $1 \in \mathcal{S}$. Thus $B_{1} A_{2}=0$. Similarly, $B_{i} A_{j}=0$ whenever $i \neq j$. 
Corollary 3.2. Let $\mathcal{A}$ be a non-commutative centrally closed prime algebra over an algebraically closed field $\mathbb{F}$. Then $\mathcal{A}$ is finite dimensional if and only if there exists an elementary operator on $\mathcal{A}$ that is a Lie (or Jordan) homomorphism but not a homomorphism.

Proof. Let $\mathcal{A}$ be finite dimensional. Since $\mathbb{F}$ is algebraically closed, we have, by the classical Wedderburn theorem, $\mathcal{A} \cong M_{n}(\mathbb{F})$ for some positive integer $n$. Moreover, $n \geqslant 2$ since $\mathcal{A}$ is non-commutative. Let $A^{\mathrm{t}}$ denote the transpose of $A \in \mathcal{A}$. The map $A \mapsto-A^{\mathrm{t}}$ (respectively, $A \mapsto A^{\mathrm{t}}$ ) is a Lie (respectively, Jordan) homomorphism of $\mathcal{A}$ that is not a homomorphism. Since every linear map on $\mathcal{A}$ is an elementary operator, this proves the 'only if' part. The 'if' part follows from Theorem 3.1.

\section{Commutativity-preserving elementary operators}

Throughout this section, $\mathcal{A}$ will be a centrally closed prime algebra over an algebraically closed field $\mathbb{F}$.

Let $\phi$ be an elementary operator on $\mathcal{A}$. We will say that $\phi$ is a standard commutativitypreserving elementary operator if there exist $A_{i}, B_{i} \in \mathcal{A}$ such that $\phi=\sum_{i=1}^{k} A_{i} M_{B_{i}}$ and $B_{i} A_{j} \in \mathbb{F} 1$ for all $i, j=1, \ldots, k$. A straightforward computation shows that such an operator indeed preserves commutativity. We remark that if $\phi$ is of length $n \leqslant k$ and $\phi=\sum_{j=1}^{n} C_{j} M_{D_{j}}$ for some $C_{j}, D_{j} \in \mathcal{A}$, then these coefficients satisfy the same condition $D_{i} C_{j} \in \mathbb{F} \mathbf{1}$. Namely, since

$$
\sum_{j=1}^{n}{ }_{C_{j}} M_{D_{j}}-\sum_{i=1}^{k}{ }_{A_{i}} M_{B_{i}}=0
$$

it follows from Lemma 2.1 and Corollary 2.2 that each $D_{j}$ belongs to the linear span of the $B_{i}$, and similarly, each $C_{j}$ belongs to the linear span of the $A_{i}$. Accordingly, $D_{i} C_{j} \in \mathbb{F} \mathbf{1}$ for all $i, j=1, \ldots, n$ follows from $B_{i} A_{j} \in \mathbb{F} \mathbf{1}$ for all $i, j=1, \ldots, k$.

There exist non-standard commutativity-preserving elementary operators.

Example 4.1. Suppose that the lower socle $\mathcal{S}$ of $\mathcal{A}$ is non-zero. Let $\mathcal{V}$ be an arbitrary non-zero finite-dimensional subspace of $\mathcal{S}$. By Litoff's theorem [3, Theorem 4.3.1] there exists an idempotent $P \in \mathcal{S}$ such that $\mathcal{V} \subseteq P \mathcal{A} P \cong M_{n}(\mathbb{F})$ for some $n \geqslant 1$. Therefore, every linear operator from $P \mathcal{A} P$ into itself is an elementary operator, and so there exist $A_{i}, B_{i} \in P \mathcal{A} P$ such that $\phi=\sum_{i=1}^{n} A_{i} M_{B_{i}}$ maps $P \mathcal{A} P$ onto $\mathcal{V}$. However, since $\phi$ clearly vanishes on $(\mathbf{1}-P) \mathcal{A} P \oplus P \mathcal{A}(\mathbf{1}-P) \oplus(\mathbf{1}-P) \mathcal{A}(\mathbf{1}-P)$ (namely, the $A_{i}$ and the $B_{i}$ lie in $P \mathcal{A} P$ ), it follows that $\phi$ maps $\mathcal{A}$ onto $\mathcal{V}$. Thus, we found an elementary operator $\phi$ on $\mathcal{A}$ whose range is $\mathcal{V}$. If we choose $\mathcal{V}$ so that it is a commutative set, then $\phi$ has a commutative range and so it trivially preserves commutativity. However, $\phi$ is not necessarily standard. The simplest concrete example is $\phi={ }_{E} M_{E}$, where $E$ is a minimal idempotent. Its range is $\mathbb{F} E$ (namely, we have assumed that $\mathbb{F}$ is algebraically closed), so it preserves commutativity. Since its length is 1 , it follows from the above observation that it is not standard unless $E=\mathbf{1}$, i.e. $\mathcal{A}=\mathbb{F} \mathbf{1}$. 
This example motivates the following definition. We will say that an elementary operator is degenerate if its range lies in the lower socle of $\mathcal{A}$. One can obtain further examples of commutativity-preserving elementary operators by combining standard and degenerate ones.

Example 4.2. Suppose that $\mathcal{A}$ contains elements $A_{1}, A_{2}, B_{1}, B_{2}$ such that $B_{i} A_{j}=\delta_{i j}$, $i, j=1,2$. In [12] algebras containing such elements were called properly infinite. A simple example is $\mathcal{B}(H)$, the algebra of all bounded linear operators on an infinite-dimensional Hilbert space $H$; there are, however, other important examples. Let $\psi_{i}, i=1,2$, be non-zero commutativity-preserving elementary operators on $\mathcal{A}$. Set $\phi_{i}={ }_{A_{i}} M_{B_{i}} \psi_{i}, i=$ 1,2 , and note that $\phi_{i}$ is also a non-zero commutativity-preserving elementary operator. Moreover, if $\psi_{i}$ is standard, then $\phi_{i}$ is standard, and if $\psi_{i}$ is degenerate, then $\phi_{i}$ is degenerate. Since $\phi_{i}(\mathcal{A}) \phi_{j}(\mathcal{A})=0, i \neq j$, it follows that $\phi=\phi_{1}+\phi_{2}$ also preserves commutativity. One can choose the $\psi_{i}$ so that $\phi$ is neither standard nor degenerate and its range is not commutative. For example, note that this is true if we take $\psi_{1}={ }_{1} M_{1}$ (i.e. $\psi_{1}$ is the identity) and $\psi_{2}={ }_{E} M_{E}$, where $E$ is a minimal idempotent.

Our main result is the following theorem.

Theorem 4.3. Let $\mathcal{A}$ be a centrally closed prime algebra over an algebraically closed field $\mathbb{F}$. If $\phi$ is a commutativity-preserving elementary operator on $\mathcal{A}$, then

$$
\phi=\phi_{\mathrm{s}}+\phi_{\mathrm{d}},
$$

where $\phi_{\mathrm{s}}$ is a standard commutativity-preserving elementary operator and $\phi_{\mathrm{d}}$ is a degenerate elementary operator.

It should be mentioned that the decomposition into a standard and a degenerate part is not unique as there exist elementary commutativity-preserving operators that are both standard and degenerate. As will be clear from the proof, we can choose $\phi_{\mathrm{s}}$ and $\phi_{\mathrm{d}}$ so that the length of $\phi$ is equal to the sum of the lengths of $\phi_{\mathrm{s}}$ and $\phi_{\mathrm{d}}$. However, we do not know whether or not they can be chosen so that $\phi_{\mathrm{d}}$ also preserves commutativity. So the problem of characterizing commutativity-preserving elementary operators on $\mathcal{A}$ remains open.

In the proof of Theorem 4.3 we will need the following elementary lemma.

Lemma 4.4. Let $\mathcal{F}$ be a linear subspace of $\mathcal{A}$. If $\phi$ is an elementary operator on $\mathcal{A}$ of length $n$, then there exist elementary operators $\phi_{1}, \ldots, \phi_{4}$ of lengths $n_{1}, \ldots, n_{4} \geqslant 0$, respectively, such that $\phi=\phi_{1}+\cdots+\phi_{4}, n=n_{1}+\cdots+n_{4}$, and all coefficients (left and right) of $\phi_{1}$ lie in $\mathcal{F}$, all left coefficients of $\phi_{2}$ lie in $\mathcal{F}$, all right coefficients of $\phi_{3}$ lie in $\mathcal{F}$, the linear span of the left coefficients of $\phi_{3}+\phi_{4}$ has trivial intersection with $\mathcal{F}$, and the linear span of the right coefficients of $\phi_{2}+\phi_{4}$ has trivial intersection with $\mathcal{F}$.

Proof. Let $\phi=\sum_{i=1}^{n} A_{i} M_{B_{i}}$. We begin with a general observation which will be needed below. Fix $i, 1 \leqslant i \leqslant n$. If $\alpha_{j}, j \neq i$, are arbitrary scalars and $A_{i}^{\prime}=A_{i}+\sum_{j \neq i} \alpha_{j} A_{j}$, $A_{j}^{\prime}=A_{j}, j \neq i, B_{i}^{\prime}=B_{i}$, and $B_{j}^{\prime}=B_{j}-\alpha_{j} B_{i}, j \neq i$, then $\phi=\sum_{i=1}^{n} A_{i}^{\prime} M_{B_{i}^{\prime}}$. Of course, an analogous change of the coefficients can be carried out with the roles of the left and right coefficients interchanged. 
There is nothing to prove when $n=1$. So, assume that $n>1$ and that the lemma is true for $n-1$. If both the linear span of the $A_{j}$ and the linear span of the $B_{j}$ have trivial intersection with $\mathcal{F}$, then $n=n_{4}$ and we are done. If not, then there is a non-trivial linear combination of the left coefficients belonging to $\mathcal{F}$, or a non-trivial linear combination of the right coefficients belonging to $\mathcal{F}$. We will only treat the second possibility here, since the proof in the other case is similar. After renumerating the coefficients, if necessary, we may assume that $B_{1}+\sum_{j \neq 1} \alpha_{j} B_{j} \in \mathcal{F}$. Applying the procedure described in the first paragraph we may even assume that already $B_{1}$ lies in $\mathcal{F}$. Now we apply the induction hypothesis to write

$$
\begin{aligned}
\phi & ={ }_{A_{1}} M_{B_{1}}+\sum_{j=2}^{n}{ }_{A_{j}} M_{B_{j}} \\
& ={ }_{A_{1}} M_{B_{1}}+\sum_{j=1}^{p}{ }_{C_{j}} M_{D_{j}}+\sum_{j=1}^{q}{ }_{E_{j}} M_{F_{j}}+\sum_{j=1}^{r}{ }_{M_{j}} M_{N_{j}}+\sum_{j=1}^{s}{ }_{P_{j}} M_{Q_{j}},
\end{aligned}
$$

where all the $C_{j}, D_{j}, E_{j}$ and $N_{j}$ lie in $\mathcal{F}$, the linear span of the $M_{j}$ and the $P_{j}$ has trivial intersection with $\mathcal{F}$, and the linear span of the $F_{j}$ and $Q_{j}$ has the same property. Of course, some of the last four terms may be the zero operator. If $A_{1} \in \mathcal{F}$, then

$$
\left(A_{1} M_{B_{1}}+\sum_{j=1}^{p} C_{j} M_{D_{j}}\right)+\sum_{j=1}^{q}{ }_{E_{j}} M_{F_{j}}+\sum_{j=1}^{r} M_{j} M_{N_{j}}+\sum_{j=1}^{s}{ }_{P_{j}} M_{Q_{j}}
$$

is a decomposition of $\phi$ into a sum of four elementary operators with the desired property. If $A_{1} \notin \mathcal{F}$, and, moreover, the linear span of $\left\{A_{1}\right\} \cup\left\{M_{j}: j=1, \ldots, r\right\} \cup\left\{P_{j}: j=\right.$ $1, \ldots, s\}$ has trivial intersection with $\mathcal{F}$, then

$$
\sum_{j=1}^{p} C_{j} M_{D_{j}}+\sum_{j=1}^{q}{ }_{E_{j}} M_{F_{j}}+\left({ }_{A_{1}} M_{B_{1}}+\sum_{j=1}^{r} M_{j} M_{N_{j}}\right)+\sum_{j=1}^{s}{ }_{P_{j}} M_{Q_{j}}
$$

is the desired decomposition of $\phi$. It remains to consider the case that $A_{1} \notin \mathcal{F}$ and that there exist scalars $\lambda_{1}, \ldots, \lambda_{r}, \mu_{1}, \ldots, \mu_{s}$ such that $A_{1}+\lambda_{1} M_{1}+\cdots+\lambda_{r} M_{r}+\mu_{1} P_{1}+\cdots+$ $\mu_{s} P_{s}$ lies in $\mathcal{F}$. Note that $N_{1}-\lambda_{1} B_{1}, \ldots, N_{r}-\lambda_{r} B_{1}$ all belong to $\mathcal{F}$. Moreover, it is easy to verify that

$$
\operatorname{span}\left\{F_{1}, \ldots, F_{q}, Q_{1}-\mu_{1} B_{1}, \ldots, Q_{s}-\mu_{s} B_{1}\right\} \cap \mathcal{F}=\{0\} .
$$

Hence, applying the change of coefficients described in the first paragraph we get

$$
\phi={ }_{A_{1}^{\prime}} M_{B_{1}}+\sum_{j=1}^{p} C_{j} M_{D_{j}}+\sum_{j=1}^{q}{ }_{E_{j}} M_{F_{j}}+\sum_{j=1}^{r}{ }_{M_{j}} M_{N_{j}^{\prime}}+\sum_{j=1}^{s}{ }_{P_{j}} M_{Q_{j}^{\prime}}
$$

with all the properties described above and with the additional property that $A_{1}^{\prime}$ belongs to $\mathcal{F}$. But as we know in this case we already have the decomposition of $\phi$ into a sum of four elementary operators with the desired properties. 
Proof of Theorem 4.3. Let $\phi=\sum_{i=1}^{n} A_{i} M_{B_{i}}$. Since $\phi$ has length $n$, by Corollary 2.2 the sets $\left\{A_{1}, \ldots, A_{n}\right\}$ and $\left\{B_{1}, \ldots, B_{n}\right\}$ are linearly independent.

We start by applying the idea from $[6]$ : since $X$ and $X^{2}$ always commute, we have $\left[\phi(X), \phi\left(X^{2}\right)\right]=0$ for all $X \in \mathcal{A}$. Note that this can be written as

$$
\sum_{i=1}^{n} A_{i} X\left(\sum_{j=1}^{n}\left[B_{i} A_{j}, X\right] X B_{j}\right)=0 \quad \text { for all } X \in \mathcal{A} .
$$

A complete linearization of (4.1) yields

$$
\begin{aligned}
\sum_{i=1}^{n} A_{i} & X_{1}\left(\sum_{j=1}^{n}\left[B_{i} A_{j}, X_{2}\right] X_{3} B_{j}+\sum_{j=1}^{n}\left[B_{i} A_{j}, X_{3}\right] X_{2} B_{j}\right) \\
+ & \sum_{i=1}^{n} A_{i} X_{2}\left(\sum_{j=1}^{n}\left[B_{i} A_{j}, X_{1}\right] X_{3} B_{j}+\sum_{j=1}^{n}\left[B_{i} A_{j}, X_{3}\right] X_{1} B_{j}\right) \\
& +\sum_{i=1}^{n} A_{i} X_{3}\left(\sum_{j=1}^{n}\left[B_{i} A_{j}, X_{1}\right] X_{2} B_{j}+\sum_{j=1}^{n}\left[B_{i} A_{j}, X_{2}\right] X_{1} B_{j}\right)=0 \\
& \text { for all } X_{1}, X_{2}, X_{3} \in \mathcal{A} .
\end{aligned}
$$

Assume first that $\mathcal{A}$ is a non-GPI algebra. We could consider the above identity as a generalized polynomial identity, but in view of our present goals it is somewhat more convenient to consider it as a generalized functional identity. Since the $A_{i}$ are linearly independent, it follows from Lemma 2.5 that for every fixed $i=1, \ldots, n$ we have

$$
\sum_{j=1}^{n}\left[B_{i} A_{j}, X_{1}\right] X_{2} B_{j}+\sum_{j=1}^{n}\left[B_{i} A_{j}, X_{2}\right] X_{1} B_{j}=0 \quad \text { for all } X_{1}, X_{2} \in \mathcal{A} \text {. }
$$

But then, since the $B_{j}$ are linearly independent, it follows, again by Lemma 2.5, that $\left[B_{i} A_{j}, X_{1}\right]=0$ for all $X_{1} \in \mathcal{A}$ and $j=1, \ldots, n$, that is, $B_{i} A_{j}$ lies in the centre of $\mathcal{A}$. Since $\mathcal{A}$ is centrally closed, this means that $B_{i} A_{j} \in \mathbb{F} \mathbf{1}$ for all $i, j=1, \ldots, n$. That is to say, $\phi$ must be standard in the non-GPI case.

We may therefore assume that $\mathcal{A}$ is a GPI algebra. Therefore, there exists a pair of dual vector spaces $\mathcal{V}$ and $\mathcal{W}$ over $\mathbb{F}$ such that $\mathcal{F}_{W}(\mathcal{V}) \subseteq \mathcal{A} \subseteq \mathcal{L}_{\mathcal{W}}(\mathcal{V})$ (see $\S 2.4$ ).

Using Lemma 4.4 (with $\mathcal{F}=\mathcal{F}_{W}(\mathcal{V})$ ) we may assume that $\phi=\phi_{1}+\cdots+\phi_{4}$, all coefficients (left and right) of $\phi_{1}$ are of finite rank, all left coefficients of $\phi_{2}$ are of finite rank, all right coefficients of $\phi_{3}$ are of finite rank, the linear span of the left coefficients of $\phi_{3}+\phi_{4}$ has trivial intersection with $\mathcal{F}_{W}(\mathcal{V})$, and the linear span of the right coefficients of $\phi_{2}+\phi_{4}$ has trivial intersection with $\mathcal{F}_{W}(\mathcal{V})$. We will consider only the case that each of the elementary operators $\phi_{1}, \phi_{2}, \phi_{3}$ and $\phi_{4}$ is non-zero since the case that some of them are zero can be treated using the same arguments (in fact, in this case the proof is even easier, for example, there is nothing to prove if $\left.\phi_{4}=0\right)$. So, we have integers $p, q$, 
$r$ such that $1 \leqslant p<q<r<n$ and

$$
\begin{aligned}
\phi_{1} & =\sum_{j=1}^{p} A_{j} M_{B_{j}}, & \phi_{2} & =\sum_{j=p+1}^{q}{ }_{A_{j}} M_{B_{j}}, \\
\phi_{3} & =\sum_{j=q+1}^{r} A_{j} M_{B_{j}}, & \phi_{4} & =\sum_{j=r+1}^{n} A_{j} M_{B_{j}} .
\end{aligned}
$$

We have to prove that for every pair of integers $s, t, r+1 \leqslant s, t \leqslant n$, there exists a scalar $\lambda_{s, t}$ such that $B_{s} A_{t}=\lambda_{s, t} I$. Equivalently, we have to prove that for every pair of integers $s, t, r+1 \leqslant s, t \leqslant n$, and for every vector $v \in \mathcal{V}$ the vectors $B_{s} A_{t} v$ and $v$ are linearly dependent. Assume that this is not the case and let $s, t$, where $r+1 \leqslant s, t \leqslant n$, be integers and let $v_{1} \in \mathcal{V}$ be a vector such that $B_{s} A_{t} v_{1}$ and $v_{1}$ are linearly independent. As mentioned in $\S 2.4$, we can find $w_{1} \in \mathcal{W}$ such that

$$
\left\langle B_{s} A_{t} v_{1}, w_{1}\right\rangle=1 \quad \text { and } \quad\left\langle v_{1}, w_{1}\right\rangle=0 .
$$

Furthermore, since the linear span of $A_{q+1}, \ldots, A_{n}$ intersects $\mathcal{F}_{W}(\mathcal{V})$ trivially, applying Lemma 2.6 we can find $v_{2} \in \mathcal{V}$ such that every non-trivial linear combination of

$$
A_{q+1} v_{2}, \ldots, A_{n} v_{2}
$$

lies outside

$$
\operatorname{Im} A_{1}+\cdots+\operatorname{Im} A_{q}
$$

Similarly, using the fact that $B \in \mathcal{A}$ has finite rank if and only if $B^{*}$ has finite rank, we infer from Lemma 2.6 that there is $w_{2} \in \mathcal{W}$ such that $\left\langle v_{2}, w_{2}\right\rangle=0$ and every non-trivial linear combination of

$$
B_{p+1}^{*} w_{2}, \ldots, B_{q}^{*} w_{2}, B_{r+1}^{*} w_{2}, \ldots, B_{n}^{*} w_{2}
$$

lies outside

$$
\operatorname{Im} B_{1}^{*}+\cdots+\operatorname{Im} B_{p}^{*}+\operatorname{Im} B_{q+1}^{*}+\cdots+\operatorname{Im} B_{r}^{*}+\operatorname{span}\left\{B_{1}^{*} w_{1}, \ldots, B_{n}^{*} w_{1}\right\} .
$$

Similarly, as above (see $\S 2.4$ ) we can find $v_{3} \in \mathcal{V}$ such that

$$
\begin{aligned}
& \left\langle v_{3}, B_{t}^{*} w_{2}\right\rangle=1, \\
& \left\langle v_{3}, B_{j}^{*} w_{2}\right\rangle=0
\end{aligned}
$$

whenever $j \neq t$, and

$$
\left\langle v_{3}, B_{j}^{*} w_{1}\right\rangle=0
$$

for every $j, 1 \leqslant j \leqslant n$.

Now, since $\left\langle v_{1}, w_{1}\right\rangle=0$ and $\left\langle v_{2}, w_{2}\right\rangle=0$, we see that the maps $S: v \mapsto\left\langle v, w_{1}\right\rangle v_{2}$ and $T: v \mapsto\left\langle v, w_{2}\right\rangle v_{1}$ satisfy $S T=0=T S$. In particular, $S, T \in \mathcal{F}_{W}(\mathcal{V}) \subseteq \mathcal{A}$ commute and so we have $\phi(S) \phi(T)=\phi(T) \phi(S)$. Since $\phi(S) v_{3}=0$ by (4.9) it follows that $\phi(S) \phi(T) v_{3}=$ 
0 . By (4.7) and (4.8) we have $\phi(T) v_{3}=A_{t} v_{1}$ and so $\phi(S) A_{t} v_{1}=0$. However, note that this together with (4.4) contradicts (4.5). This proves that $\phi_{4}$ is a standard commutativitypreserving elementary operator. Now set $\phi_{\mathrm{s}}=\phi_{4}$ and $\phi_{\mathrm{d}}=\phi_{1}+\phi_{2}+\phi_{3}$, and note that $\phi_{\mathrm{s}}$ and $\phi_{\mathrm{d}}$ have the desired properties.

Theorem 4.3 and Example 4.1 together yield the following corollary.

Corollary 4.5. Let $\mathcal{A}$ be a non-commutative centrally closed prime algebra over an algebraically closed field $\mathbb{F}$. Then there exists a non-standard commutativity-preserving elementary operator on $\mathcal{A}$ if and only if $\mathcal{A}$ has a non-zero lower socle.

Thus, if the lower socle of $\mathcal{A}$ is zero (i.e. if $\mathcal{A}$ is not a GPI algebra), then every commutativity-preserving elementary operator $\phi$ on $\mathcal{A}$ is standard (i.e. $\phi=\phi_{\mathrm{s}}$ ). In another extreme when $\mathcal{A}=M_{n}(\mathbb{F})$, Theorem 4.3 does not provide any useful information since every linear operator on $\mathcal{A}$ is automatically degenerate. However, in this case the result from $[\mathbf{2 1}]$ mentioned above gives the definitive conclusion (at least when the characteristic of $\mathbb{F}$ is 0 ). So the most interesting case is the one where $\mathcal{A}$ is an infinitedimensional algebra with non-zero lower socle (i.e. $\mathcal{A}$ is a GPI but not a PI algebra). Here, Theorem 4.3 gives some basic, but not yet complete, information.

\section{References}

1. S. A. Amitsur, Generalized polynomial identities and pivotal monomials, Trans. Am. Math. Soc. 114 (1965), 210-226.

2. P. Ara And M. Mathieu, Local multipliers of $C^{*}$-algebras (Springer, 2003).

3. K. I. Beidar, W. S. Martindale III And A. V. MikhaleV, Rings with generalized identities (Marcel Dekker, 1996).

4. K. I. Beidar, M. Brešar, M. A. Chebotar and Y. Fong, Applying functional identities to some linear preserver problems, Pac. J. Math. 204 (2002), 257-271.

5. K. I. Beidar, M. Brešar, M. A. Chebotar and W. S. Martindale III, On Herstein's Lie map conjectures, III, J. Alg. 249 (2002), 59-94.

6. M. BREŠAR, Commuting traces of biadditive mappings, commutativity-preserving mappings and Lie mappings, Trans. Am. Math. Soc. 335 (1993), 525-546.

7. M. BrešAr, Functional identities: a survey, Contemp. Math. 259 (2000), 93-109.

8. M. BREŠAR, Commuting maps: a survey, Taiwan. J. Math. 8 (2004), 361-397.

9. M. BREŠAR AND D. EREMITA, The lower socle and finite rank elementary operators, Commun. Alg. 31 (2003), 1485-1497.

10. M. BREŠAR AND P. ŠEMRL, On locally linearly dependent operators and derivations, Trans. Am. Math. Soc. 351 (1999), 1257-1275.

11. M. A. Chebotar, On generalized functional identities on prime rings, J. Alg. 202 (1998), 655-670.

12. H. G. Dales, N. J. Laustsen and C. J. Read, A properly infinite Banach *-algebra with a non-zero, bounded trace, Studia Math. 155 (2003), 107-129.

13. I. N. Herstein, Lie and Jordan structures in simple, associative rings, Bull. Am. Math. Soc. 67 (1961), 517-531.

14. L. HuA, A theorem on matrices over an $s$-field and its applications, J. Chin. Math. Soc. 1 (1951), 110-163.

15. N. JaCOBSOn, Lie algebras (Interscience, New York, 1962).

16. P.-H. LeE, J.-S. Lin, R.-J. WAng And T.-L. Wong, Commuting traces of multiadditive mappings, J. Alg. 193 (1997), 709-723. 
17. W. S. Martindale III, Prime rings satisfying a generalized polynomial identity, J. Alg. 12 (1969), 576-584.

18. W. S. Martindale III, The extended center of coproducts, Can. Math. Bull. 25 (1982), 245-248.

19. M. Mathieu, Rings of quotients of ultraprime Banach algebras, With applications to elementary operators, Proc. Centre Math. Analysis Austral. Nat. Univ. 21 (1989), 297317.

20. R. Meshulam And P. ŠEmrl, Locally linearly dependent operators, Pac. J. Math. 203 (2002), 441-459.

21. M. Omladič, H. Radjavi and P. Šemrl, Preserving commutativity, J. Pure Appl. Alg. 156 (2001), 309-328.

22. W. WAtKins, Linear maps that preserve commuting pairs of matrices, Linear Alg. Applic. 14 (1976), 29-35.

23. G. A. Willis, Ultraprime group algebras, Proc. Centre Math. Analysis Austral. Nat. Univ. 21 (1989), 345-349. 